\title{
Micro-grid Remote Servo System Based on the World Wide Web
}

\author{
Yizhi Ye*1,2,a , Nan Lvc, Jiale Liü,d \\ ${ }^{1}$ Shanghai Jiao Tong University department of Automation, \\ Shanghai 200240, China. \\ ${ }^{2}$ Shanghai Electric Group CO.LTD Central Academe, \\ Shanghai 200240, China. \\ ayeyz@shanghai-electric.com, \\ bliujiaojiaopingguo@163.com
}

\begin{abstract}
In order to facilitate monitor and manage various distributed generations in micro-grid, eliminate and discover malfunction of micro-grid, a micro-grid remote servo system based on The World Wide Web was designed. Discussing micro-grid's remote monitoring system functional structure and database system. The system can realize remote micro-grid monitoring under the internet environment. Finally the micro-grid remote servo interface was designed.
\end{abstract}

Keywords-micro-grid; remote servo; database; internet

\section{INTRODUCTION}

The real-time monitoring and timely diagnosis can meet the requirements for the safety of micro-grid. Monitoring personnel can be like in the field by using remote monitoring technology. It can eliminate restrictions due to the time and place to micro-grid management in this way[1].

\section{A. General structure of remote servo system}

The World Wide Web micro-grid remote servo system is based on SVG(Scalable Vector Graphics) and Ajax (Asynchronous JavaScript and XML) technology. It can realize the wiring diagram of the system manual configuration and display data and operation state of power equipment. Users do not need to install any client software, and can use the browser for a visit directly. Firstly the

\author{
Jiaojiao Liu ${ }^{3, b}$ \\ ${ }^{3}$ Shanghai Dianji University, Shanghai 200240, China. \\ 'lvnan@shanghai-electric.com, \\ dliuj15@shanghai-electric.com
}

server starts www server. Secondly the client makes an HTTP request to the server. Finally the client operating management interface back to the customer in the form of web pages.

Servo system backstage uses an application server and a database server, and can be extended based on the number of users to access. A firewall was used to protect server on the network. Ordinary datum can be transmitted via Http and sensitive datum can be transmitted via Https. Data transmission flow chart was shown in Figure 1.

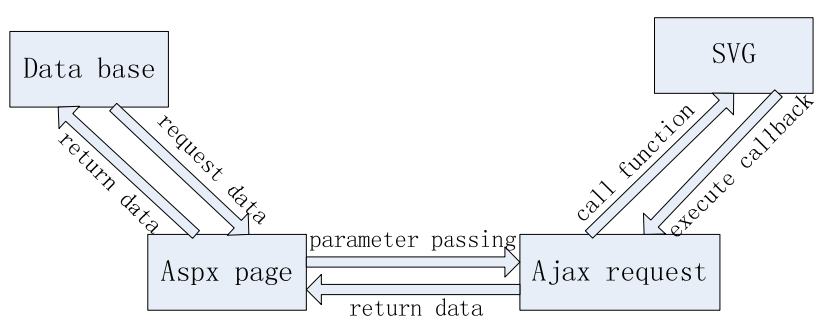

Figure 1. Data transmission flow chart

Servo system server program interact with the client through Web API. It uses the JSON data format for transmission when interacting. Specific contents are as follows: 


\begin{tabular}{|c|c|c|c|}
\hline Name of API & $\begin{array}{c}\text { function } \\
\text { declaratio } \\
n\end{array}$ & invocation example & return value example \\
\hline $\begin{array}{l}\text { Login(string } \\
\text { user_Login, string } \\
\text { user_Password) }\end{array}$ & User login & $\begin{array}{l}\text { Login(“Z002tjrn”,“123456 } \\
\text { ”) }\end{array}$ & $\begin{array}{l}\text { Return: } \\
\text { \{“status”: “200”, “msg”: “The request has succeeded”,“rt”: } \\
\text { [\{“User_Row”:4,“User_Login”:“Z002tjrn, } \\
\text { “User_Password":“e10adc3949ba59abbe56e057f20f883e”,“User_Name”:“Administrat } \\
\text { or”, “Region_row”:0\} } \\
\text { ] } \\
\quad \text { \} }\end{array}$ \\
\hline $\begin{array}{l}\text { GetProjectList(strin } \\
\text { g user_Row) }\end{array}$ & $\begin{array}{l}\text { Read the } \\
\text { list of } \\
\text { projects }\end{array}$ & GetProjectList(4) & 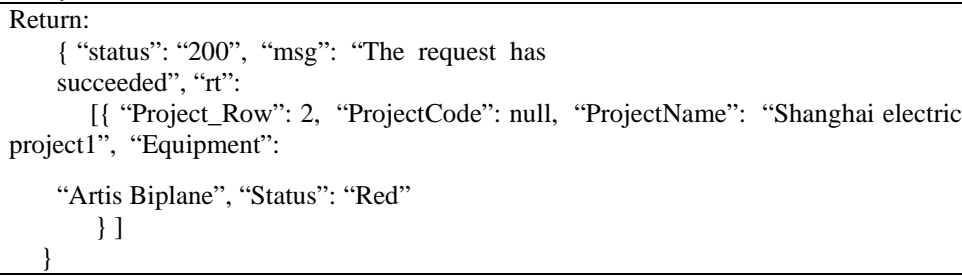 \\
\hline $\begin{array}{l}\text { SearchProject } \\
\text { (string user_Row, } \\
\text { string searchFilter, } \\
\text { string searchString) }\end{array}$ & $\begin{array}{l}\text { Search } \\
\text { project }\end{array}$ & $\begin{array}{l}\text { SearchProject(4, } 1 \text {, string } \\
\text { searchString) }\end{array}$ & $\begin{array}{l}\text { Return: } \\
\text { \{ "status”: “200”, “msg”: “The request has succeeded”, "rt": } \\
\text { [ \{ "Project_Row”: 2, "ProjectCode”: null, "ProjectName”: "Shanghai electric } \\
\text { project 2", "Equipment”: “Artis Biplane”, "Status”: "Red” } \\
\text { \} ] } \\
\text { \} }\end{array}$ \\
\hline
\end{tabular}

Running topology structure of server hardware is shown in figure 2.

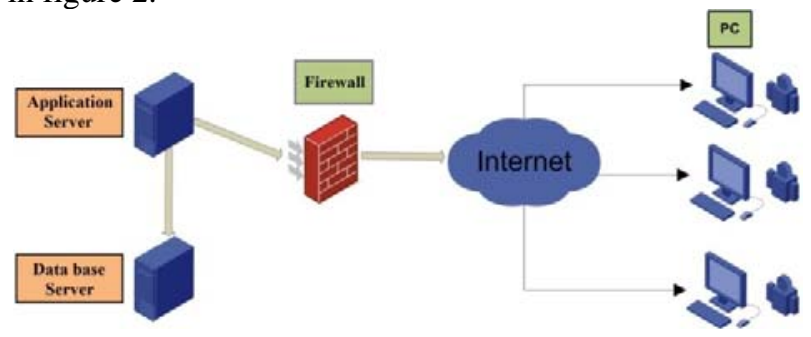

Figure 2. Running topology structure of server hardware

\section{B. Micro-grid remote customer database}

Remote customer database provides uniform data integration service management implementation methods, and support heterogeneous data in project management. a standardized service interface for its datum was provided by remote customer database. It also provides uniform data format and contract according to different terminal types. Different access requests only need to call the customer service database and in this way it can provides a remote interface to interact. Implementation details of the behind needn't to be cared about. Remote database service is also responsible for the routine maintenance of the data in order to provide stable service guarantee.

\section{Structure of remote customer database}

Not only requirements of the general cloud database software and hardware but also special demand datum need to be uploaded to the micro-grid expert knowledge base using the solutions provided in this article. The goal here was to give knowledge base time for calculation, judgment and treatment. The solution also supports the future of database system software upgrade or replace, without adding new costs. Potential future compatibility was maximum found out meet customer in addition to meet customers' current stage requirements.

In this paper, the cloud database is the set of all micro-grid data and contains a variety of ingredients, including table, record, field, index, etc. For modular development, and to facilitate future upgrades, management. The database can be divided clearly into eight kinds of table structure depending on different purposes, including project related table structure, management authority table structure, event related table structure, control table structure, four control table structure, types of related table structure, parameter table structure and communication of related table structure. In this paper, we give relevant control table structure and communication related table structure. Relevant control table structure was shown in figure 3 and communication related table structure was shown in figure 4 . 


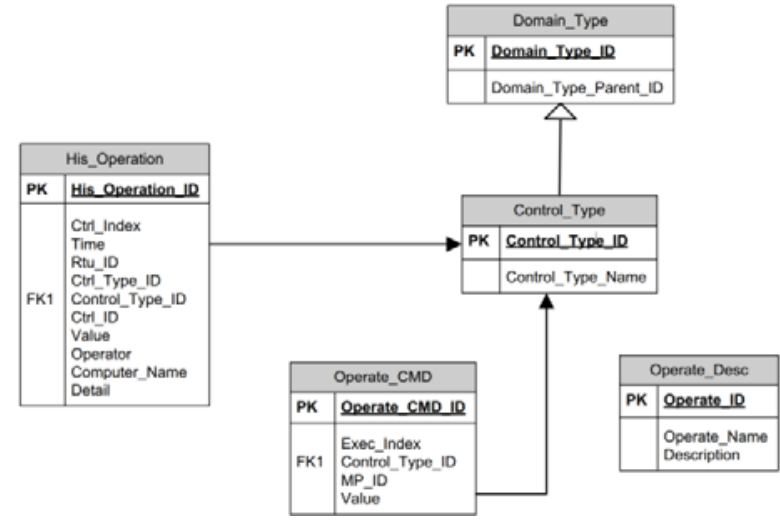

Figure 3. Relevant control table structure

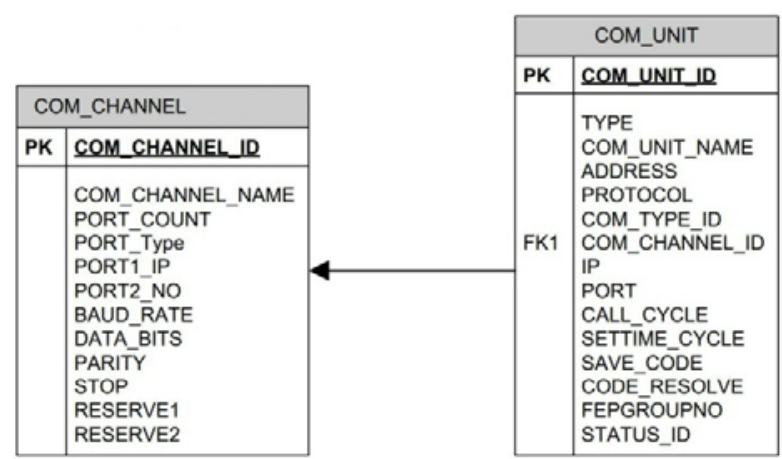

Figure 4. Communication related table structure

\section{Micro-grid remote servo man-machine interface}

Micro-grid remote servo system based on The World Wide Web can complete online micro-grid four remotes (telecontrol, remote message and remote adjustment) monitoring and control. Micro-grid real-time operation, query of history data, expert knowledge base and energy management call function can realize by remote monitoring [4]. Figure 5, 6, and 7 which respectively represent energy conservation and emissions reduction interface, real-time monitoring interface and historical event interface.

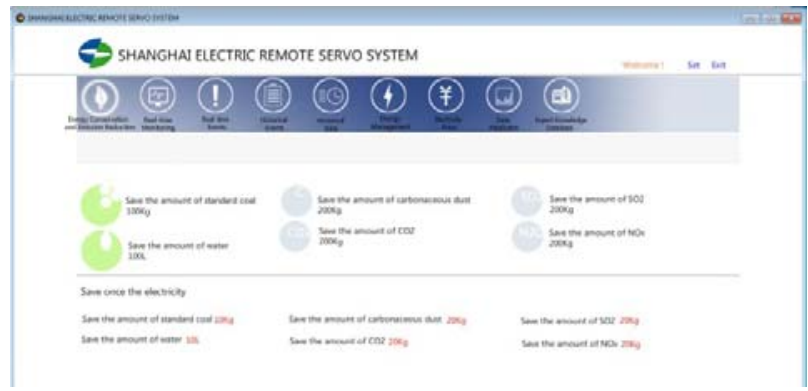

Figure 5. Energy conservation and emissions reduction interface

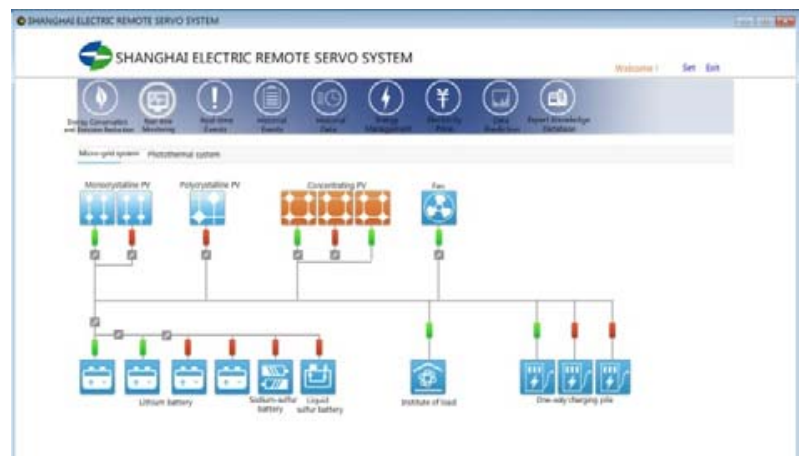

Figure 6. Real-time monitoring interface

\section{SUMMARY}

Remote servo system can blend client, network and database technologies together to offer a good solution for remote client products and services. The whole system can complete online micro-grid four remotes (telecontrol, remote message and remote adjustment) monitoring and control. Since the structure is not directed against the specific system, it has the versatility[3]. If the remote real-time monitoring structure want to be used in the occasions with higher requirements, the speed of Web Service must to be improved.

\section{REFERENCES}

[1] Sun jun. Zhu xiaoyong and Zhu zhengming: Network remote monitoring system based on Internet. Electric power automation equipment Vol. 23 (2003), p. 49-51.

[2] Guan shouping. Bao fang and Liu nian: Internet-based remote client service system architecture and key technology. Science and Technology Plaza Vol. 6 (2005), p. 8-12.

[3] Zhang yu. Liu feng: The design and implementation of a remote monitoring system structure. West southwest normal university journal Vol. 31 (2006), p. 105-109.

[4] Yang zhongbo. Tang luxin and Chen hui: Based on the implementation of embedded web remote water quality monitoring system Vol. 1 (2010), p. 9-11. 